“(C) 2015 IEEE. Personal use of this material is permitted. Permission from IEEE must be obtained for all other uses, in any current or future media, including reprinting/republishing this material for advertising or promotional purposes, creating new collective works, for resale or redistribution to servers or lists, or reuse of any copyrighted component of this work in other works." 


\title{
Augmented Reality Based Monitoring of the Remote-Lab
}

\author{
Zenon Chaczko ${ }^{1}$, Wael Alenazy ${ }^{2}$, Lucia Carrion ${ }^{1}$, Amy Tran ${ }^{1}$ \\ ${ }^{1}$ Faculty of Engineering and Information Technology \\ University of technology, Sydney (UTS), Sydney, NSW, Australia \\ E-mail: \{Zenon.chaczko, Lucia.C.Carrion\}@uts.edu.au, Amy.Tran@alumni.uts.edu \\ ${ }^{2}$ Self-Development Skills Department, Preparatory Year Deanship \\ King Saud University (KSU) \\ Riyadh, The Kingdom of Saudi Arabia \\ E-mail: Walenazy@ksu.edu.sa
}

\begin{abstract}
Augmented Reality technology approach has been being adopted within the education sector. The advanced technology tools in many classes have the potential changed of users' attitudes toward the pedagogical and psychological objectives and goals. Moreover, augmented reality has not elicited so much attention within the corridors of education sector as it is now. In order to improve the interactive effectiveness in the smart classroom environment, there is a demand to tailor the innovation technology and align it with every changing requirements and capabilities of various users. Consequently, the educators are increasingly finding augmented reality suitable for deployment in education. In this paper, a project shows how Augmented Reality utilised with overlay Smart-Grid can support the learning process in attractive methods for monitoring events of captured scenes in remote-lab such as video stream, Web-link from smart devices' camera.
\end{abstract}

Keywords—augmented reality; smart grid; remote-lab.

\section{INTRODUCTION}

Augmented Reality (AR) allows for the establishment of collaborative environments through teacher's and students' interaction within virtual objects leading to the creation of various interactive scenarios in the classroom. Virtual reality refers to the combination of various display and devices' interface which results from immersive overlay interactive computer-generated environment. In addition, the Mixed of reality refers to the merger of virtual overlay objects with on the real scene [1]. Although in terms of acceptance technology, a recent model has been appeared and showed that because of some smart technologies features, users are capable to function those devices easily [2]. Hence, AR as a smart innovation system is being incorporated in the learning and teaching processes to achieve the pedagogical objectives and goals demand. The AR system in classes allows the students to locate the students to discover and explore the virtual materials as they are in real through the use of overlaid scenes[3].

In this paper, an experiment has applied for utilising augmented reality (AR) elements within UTS Remote-Labe. The aims of the study are: firstly is to monitor captured (selected) of a scene for studying purposes, such as a video stream from an iPad camera, within the novel idea 'a smart grid layout'. The process of the experiment has toolkits to leverage functionalities. Secondly, the significant objective is also to investigate the capabilities AR frameworks and Software Development Kits (SDK) for determining and selecting particular cell(s). Furthermore, the main focus of toolkits is to provide a simple way to overlay an image or animation over a video stream. Hence, a number of prototypes have been utilised in different AR toolkits, and ultimately the project has optimised an open source image-processing framework 'OpenCV'. The case study has experimented with the use of the Metaio AR SDK, Wikitude SDK and Total Immersion's D'Fusion SDK. Moreover, due to image-processing tools, an OpenCV is required because of its characteristics. As a result, the function of monitoring and selecting of this project have succeeded and meet the study's gaols. The first section, the paper will show the use of AR into education process and its advantages. The second section introduces the case study that reveals the mixture of $\mathrm{AR}$ and the novel smart-grid feature. The final section includes conclusion and recommendation.

\section{AR IN THE CLASSROOM}

Augmented reality (AR) shifts the meaning of converting the virtual materials into genuine classroom environment by using spectacular technological techniques. In other words, it is the technology that schemes digital pedagogical materials onto real objects. The use of $\mathrm{AR}$ and its associated technologies in education permits both instructors and students to experience the virtual interaction in real time [4]. Real time learning, sharing of information and pedagogical interactions is a new concept that is in line with the level of civilisation in the world today. It is flexible system application that can be used and deployed already in available technological platforms and devices such as personal computers, laptops, tablets and smart phones. These devices are commonly used by students and educators alike. Moreover, they are easy to use and therefore suitable for suitable for students from various age groups and education level. Billinghurst (2002) pointed out that a smooth transmission between reality and virtuality will enhance and create a new experience. However, the integration into any system needs to be through an intermediate interface to allow for collaboration. Hence, many of AR and other tangible user interface system have been deployed and introduced in classes equipped specially for visualisation purpose. In contrast, in 
ordinarily equipped classrooms with no special instruments. Instructors are responsible for conducting lectures and activities by placing course books in digital form and using computers facilities. Learners follow instructor's guidelines while receiving prompts through slides shows, handouts or whiteboard. However, the equipped AR classrooms are relatively expensive making it difficult for their integration into the high collaborative environment that they must to be. Preexisting teaching using both virtual and real time materials or re-planned activities in class is still fairly structured and limited for use with AR [5]. In the education sector, many educators attempt to teach and help their students to acquire the curriculum in an enthusiasm style. By using advanced interactive technology in education, the classrooms are likely to be the favourite place for teaching and learning process. Nowadays, Augmented reality has shaped a new interaction method for many sectors other than education. Consequently, a new interaction level has been rapidly increased by introducing AR. The new advanced technologies within the classroom facilities play a vital role in elevating the motivation levels amongst student while they are taught which is vital when it comes to attaining learning outcomes and other pedagogical outcomes objectives

\section{A. Advantages of AR to Education}

With its three dimensions and real time interactivity capabilities, AR has key advantages over the traditional classroom which has seen it revolutionise the learning process. It uses some of the commonest technologies in today's world: computing and mobile telephony technologies. Most of the devices suing these technologies are very portable and therefore permits learning anywhere. That is, AR permits learning on the go. Such convenience enables students to learn with relative ease without the need to be confined within the walls of a traditional classroom setting. It allows for educators to disseminate content to the students from the remote location. It is an important $\operatorname{cog}$ in the wheel of e-learning whose popularity has increased with the advent computing technology and the internet, such as Remote-Lab. The educators and students have a platform where they can interact with the Remote-Lab in real time; doing the experiments.

Shelton and Hedley (2002) in their study of the impact of $\mathrm{AR}$ in the education sector found that there was a positive impact among undergraduate student using $A R$ in geography lesson. The study targeted over thirty students and found that there was a significant improvement in student understanding when AR was used during the learning process. They also established that there was a reduction in student misunderstanding of the various lesson concepts [3]. This is because not only does AR improves the motivational levels amongst learners it also improves the creativity and innovativeness amongst learners. Moreover, AR superimposes and enhances information on the real world elements. Such contextual information which combines visual and sensory information results in improved cognitive skills and enhances associative learning [5]. AR provides learners with a friendly interactive interface which is rich in knowledge leading to high motivation. It is modern and most learners and educators in today's world can easily identify with it leading to high learning performance [5].

\section{B. AR Based Visualisation and Interaction}

Visualisation system opens new dimensions of human computer interaction by providing accessibility and user interface to all areas. AR provides interaction between the devices and users that leads to a decrease tangible interaction. On the other hand, in ordinary classroom, classrooms have limitations and allow less functionality in the workplace to be accomplished. However, technologies that provide and advanced technique, such as gesture and speech interaction, help our daily activities to interact with real world objects in more natural way [6]. Thus, the functionalities of realitybased-interface provide the next generation interface to interact simultaneously with real and augmented environment [6]. To address all obstacles in traditional classes or equipped classes, the augmented reality technique plays a crucial role in enriching and boosting learners to achieve their goals efficiently.

Visualising things and creation of a big loophole in understanding are caused by teaching situation and demands for many concept, such as physics, chemistry and biology [6]. AR tries to solve these issues by combining real and overlaying information and visualising scenes in a proper way to understand with the interaction function. Nevertheless, there is still need to improve the interaction method in a way that will provide the reality of interaction meaning with objects [7]. Supporting the AR technique needs to be proved by applications and tangible novel devices, such as smart overlay grid. Most of the applications do not support gesture-base direct manipulating of the augmented scene that is responsible for user interaction with objects for more real interaction efficiency [7]. Therefore, our study has come up with solutions that enable AR interaction which includes new methodology, deploying user's smart selection tool in both virtually and the possibility of physical interaction with objects that will be shown to the end-user.

According to Dunleavy (2009), a simulation study showed that technology-mediation within AR technique helps with interaction and collaboration within the highly engaging environment in which teachers and students operate. Although the AR simulation system provided prospective added value to the learning and teaching process, it showed some technical, manageability and cognitive challenges [8]. It is expected that AR technology will continue to progress to deliver high quality multimedia-interaction towards more powerful for mix-reality.

\section{AR - SMARTGRID FOR EDUCATIONAL REMOTE-LAB MONITORING (CASE STUDY)}

The case study utilises augmented reality (AR) elements to select and monitor captured of scenes for studying purposes, such as a video stream from an iPad camera, within the novel idea a smart grid layout. The process of the experiment that has taken is to develop application and investigate existing AR 
toolkits to leverage functionalities. Moreover, the significant objective is to investigate the capabilities AR frameworks and Software Development Kits (SDK). The main focus of toolkits is to provide a simple way to overlay an image or animation over a video stream. So, after a number of prototypes utilising different AR toolkits; ultimately the project has optimised an open source image-processing framework 'OpenCV'. The case study has been experimented with the use of the Metaio AR SDK, Wikitude SDK and Total Immersion's D'Fusion SDK. As a result, the monitoring functionality of this application required image-processing tools and because of that OpenCV seems to be sufficient due to its characteristics.

\section{A. The Study's Rationale}

In an educational context, there are many situations where AR can be applied. In studies by Montero et al. [10] AR technology was leveraged as a means to overcome the current limitations of Classroom Communication Systems (CCS), and thus allowing teachers to obtain a personal feedback from students in the class [10]. The communication system was utilised to allow participating students to signal whether they understand the material being presented to them by the lecturer in a non-intrusive manner. The flow of the lecture was not hindered and this method also overcame the issue where students are too shy to speak up or interrupt in the classroom [10]. Typically, a classroom communication system like this also allows the lecturer to gauge which students are not paying attention if they are not providing regular feedback. Another approach to determine attentiveness is to monitor the classroom for facial expressions and body language. This is a possible extension to the monitoring application, which would require advanced image processing and machine learning functionalities.

A monitoring application can also be applied to analysing data such as graphs and gauges. For example, a researcher is required to observe an experiment with graph and meter outputs from sensor data. He needs to record events where there are spikes in the output. The application can be employed to automate this process and all that is required of the researcher is some initial configuration.

\section{B. Architecture and Design of the AR SmartGrid}

In case-study, it has been chosen iPad running iOS 7.1.1 for testing environment for the $\mathrm{iOS}$ application. In addition, Xcode 5.1 which is the Integrated Development Environment (IDE) required developing on the iOS platform. The IDE is provided free from the App Store by Apple and comes with all the essential framework and libraries required to start developing applications. Having one central IDE makes it easy for third party frameworks, such as AR Software Development Kits (SDK), to import to iOS projects. Moreover, Objective-C, Objective-C++ and $\mathrm{C}++$ have also been selected for programming languages that used to develop AR SmartGrid because Objective- $\mathrm{C}$ is the default language in the iOS framework. Objective-C++ combines $\mathrm{C}++$ elements with Objective- $\mathrm{C}$ to extend the language allowing the use of $\mathrm{C}++$ features. $\mathrm{C}++$ has been utilised for the complex image processing functionality because of the better performance and also allows easy interfacing with the OpenCV SDK, which is written in $\mathrm{C}++[11]$.

OpenCV is an open source library for computational image processing written in optimized $\mathrm{C} / \mathrm{C}++[12]$. The library has a very active support community and extensive documentation. The source code is freely available on GitHub and supports Windows, Linux/Mac, Android and iOS.

\section{Architectural Model of AR SmartGrid System}

The solution that has been developed is a prototype application that utilises monitoring grid overlay functions. The AR application, called SmartGrid, has been built on the iOS platform for iPad devices and interfaces with a corresponding also from Pebble Smart Watch application. Figure 1 presents the high level architecture of the AR SmartGrid application. The Input Handler takes either a video stream feed or a website as the scene to be displayed. So, the Smart Grid provides an overlay of the smart Cells of the grid. If a Cell is selected, the Motion Detector monitors the Cell. The Motion Detector utilises the image processing functionalities of the OpenCV SDK. If motion is detected, the Cell generates an Event, which is displayed on the screen. Setting and event logs are persisted by the local application database.

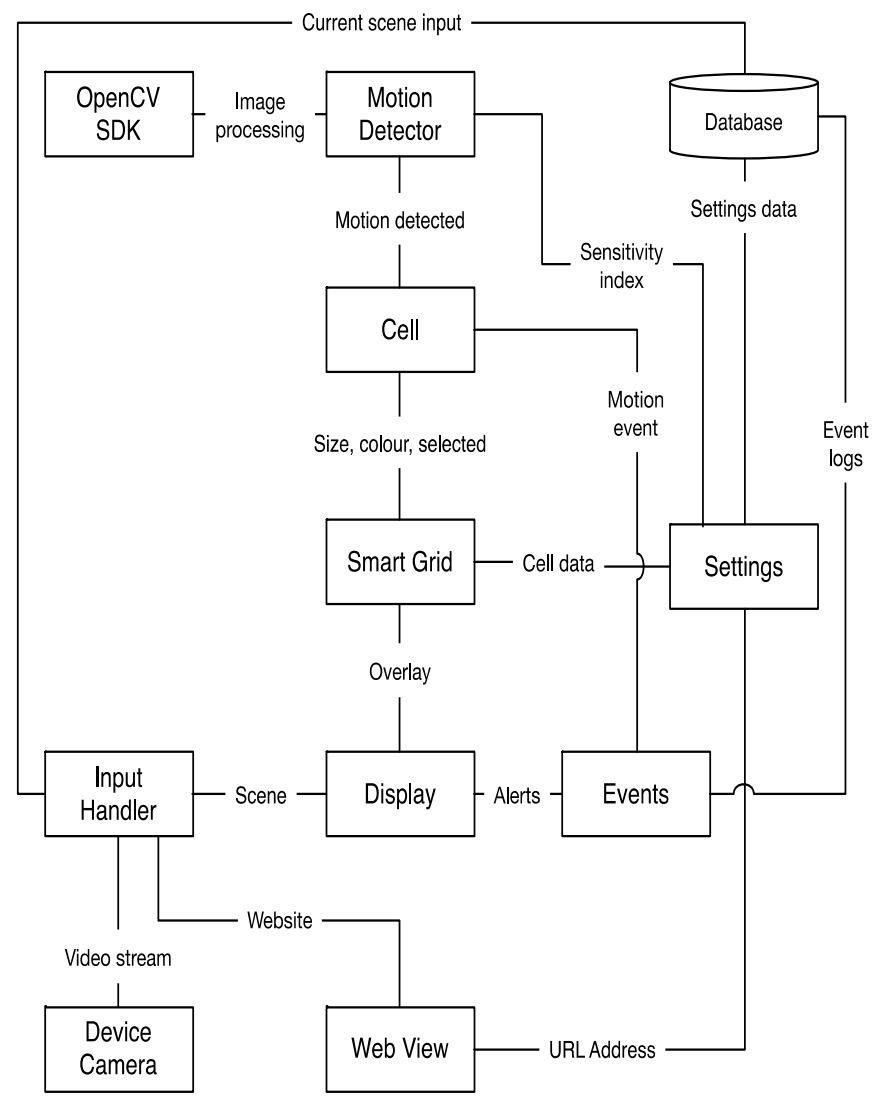

Fig. 1. High level architecture of AR Smart Grid application 


\section{Implementation of AR SmartGrid System}

The functionality of the AR SmartGrid app is that it monitors selected cells for movements or deviations that are selected by students or instructor while they are in the session remotely. The monitored scene can either be configured for a video stream from the iPad device's camera or a web view of any website (i.e. UTS Remote Labs facility [13]). The grid for monitoring activities or events can be formed as free (Fig. 2) or fixed (Fig. 3 and Fig. 4). In a free grid placement mode, monitored cells are organised by dragging and dropping cells freely onto the canvas and in the fixed grid mode cells are laid out evenly according to a configurable number of rows and columns.

When an event or status change is detected in a selected cell by touching screen or through Pebble Smart Watch for selecting, an alert popover will overlay the screen. A log of the event, including a screenshot and timestamp, is registered.

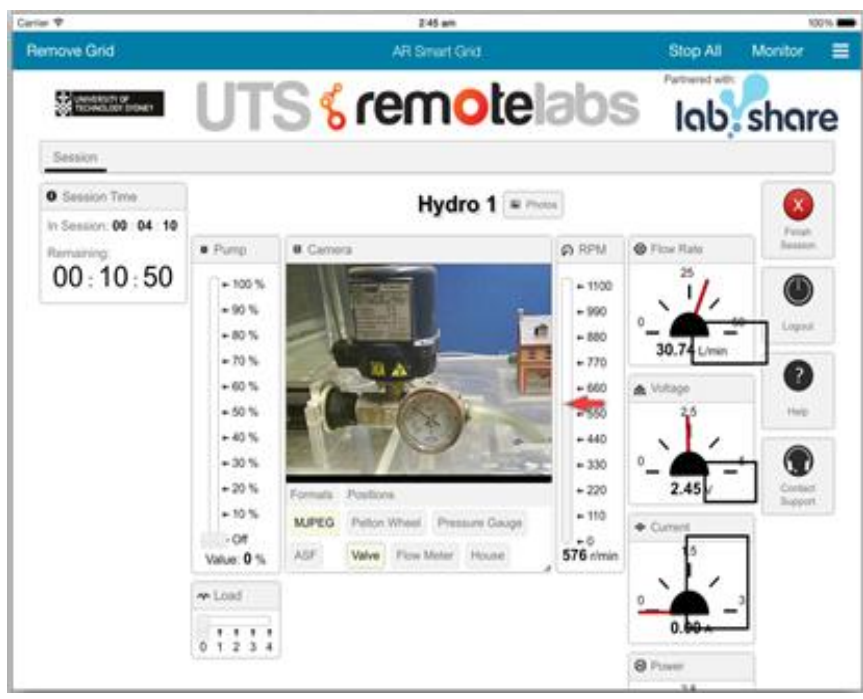

Fig. 2. SmartGrid's free grid mode by dragging and dropping cells running the UTS Remote Lab's Hydro experiment [13].

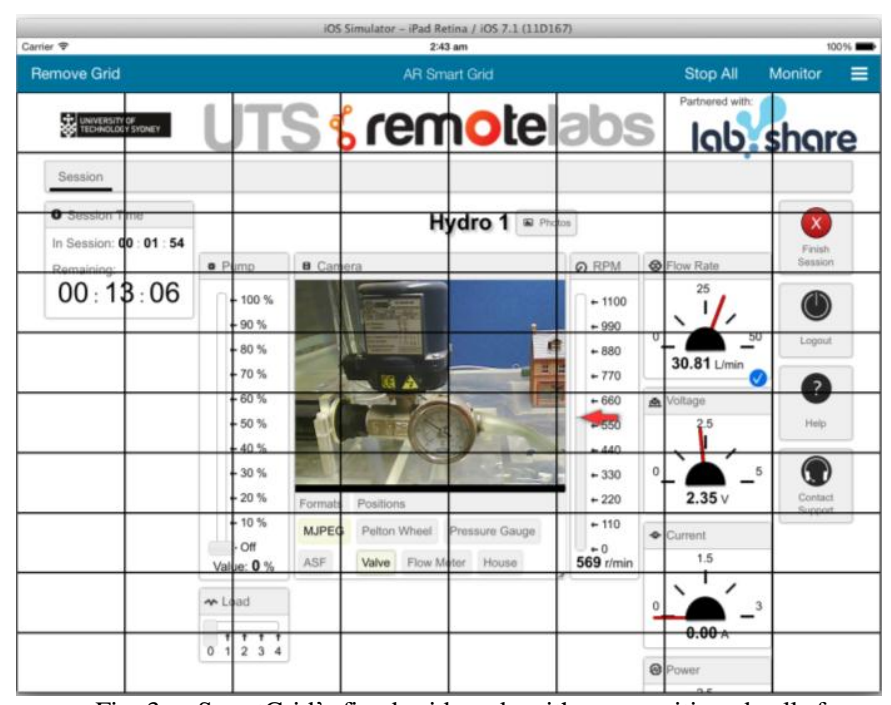

Fig. 3. SmartGrid's fixed grid mode with user positioned cells for threshold running the UTS Remote Lab's Hydro experiment [13].

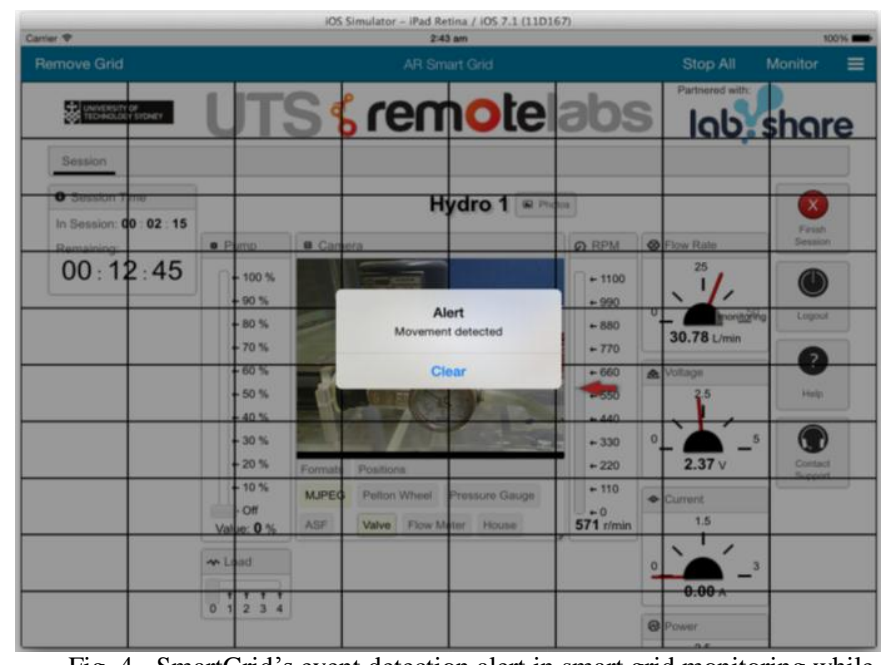

Fig. 4. SmartGrid's event detection alert in smart grid monitoring while using the UTS Remote Lab's Hydro experiment [13].

\section{CONCLUSION}

Augmented Reality as an assistance tool has been increasingly integrated within the learning environment to support learning process and create the meaningful of interactive learning platform. The integration helps to improve knowledge acquisition and retention among learners. Through the advantages, AR can play a key role in raising motivation levels in the education environment by showing pedagogical materials in interactive and enthusiastic style.

For the enhancement of particular study room such as remote-lab, a case study has been taken place to adapt AR within overlay SmartGrid into remote-lab for monitoring and selecting scene of events. The development of this prototype application associated with the advantages of using ARSmartGrid in remote-labs has demonstrated the possible to leverage existing technology for the purpose of monitoring and controlling. There is also opportunity to extend the monitoring capabilities of the application to include face detection, for example, and other features that could be possible to enhance the learning process for future demand. The use of AR technology in the context of education is still being explored, but has yielded promising results so far.

\section{REFERENCES}

[1] Z. Pan, A. D. Cheok, H. Yang, J. Zhu, and J. Shi, "Virtual reality and mixed reality for virtual learning environments," Computers \& Graphics, vol. 30, pp. 20-28, 2006

[2] Z. Chaczko and W. Alenazy, "The Extended Technology Acceptance Model and the Design of the 21st Century Classroom," in 2 nd AsiaPacific Conference on Computer Aided System Engineering-APCASE 2014.

[3] R. Freitas and P. Campos, "SMART: a SysteM of Augmented Reality for Teaching 2 nd grade students," in Proceedings of the 22nd British HCI Group Annual Conference on People and Computers: Culture, Creativity, Interaction-Volume 2, 2008, pp. 27-30.

[4] S. Cuendet, Q. Bonnard, S. Do-Lenh, and P. Dillenbourg, "Designing augmented reality for the classroom," Computers \& Education, vol. 68, pp. 557-569, 2013.

[5] D.-R. Chen, M.-Y. Chen, T.-C. Huang, and W.-P. Hsu, "Developing a Mobile Learning System in Augmented Reality Context," International Journal of Distributed Sensor Networks, vol. 2013, 2013. 
[6] S. Prasad, S. K. Peddoju, and D. Ghosh, "Mobile augmented reality based interactive teaching \& learning system with low computation approach," in Computational Intelligence in Control and Automation (CICA), 2013 IEEE Symposium on, 2013, pp. 97-103.

[7] W. H. Chun and T. Höllerer, "Real-time hand interaction for augmented reality on mobile phones," in Proceedings of the 2013 international conference on Intelligent user interfaces, 2013, pp. 307-314.

[8] M. Dunleavy, C. Dede, and R. Mitchell, "Affordances and limitations of immersive participatory augmented reality simulations for teaching and learning," Journal of Science Education and Technology, vol. 18, pp. 722, 2009.

[9] C. Dede, "Immersive interfaces for engagement and learning," science, vol. 323, pp. 66-69, 2009.
[10] A. Montero, T. Zarraonandia, I. Aedo, and P. Díaz, "Uses of Augmented Reality for Supporting Educational Presentations," in Advanced Learning Technologies (ICALT), 2013 IEEE 13th International Conference on, 2013, pp. 426-428.

[11] P. Sholtz, "How To Make An Augmented Reality Target Shooter Game With OpenCV: Part 3/4," in RAYWENDERLICH TUTORIALS FOR DEVELOPERS \& GAMERS, ed, 2014.

[12] OpenCV. (2014). Open Source Computer Vision. Available: http://www.opencv.org.

[13] Remote Lab at UTS, http://www.uts.edu.au/about/faculty-engineeringand-information-technology/what-we-do/facilities-and-services/remote. 